Cite this: Soft Matter, 2014, 10, 2982

Received 8th October 2013

Accepted 13th January 2014

DOI: $10.1039 / c 3 s m 52604 f$

www.rsc.org/softmatter

\section{Direct three-dimensional imaging of polymer- water interfaces by nanoscale hard X-ray phase tomography $\dagger$}

\author{
Yin Cheng, ${ }^{\text {ad }}$ Heikki Suhonen, ${ }^{\mathrm{b}}$ Lukas Helfen, ${ }^{\text {ab }}$ Junsheng $\mathrm{Li}^{\text {,e }}{ }^{\mathrm{F}}$ Feng $\mathrm{Xu}^{\mathrm{a}}{ }^{\mathrm{a}}$ \\ Michael Grunze, $\star^{e}$ Pavel A. Levkin*ce and Tilo Baumbach ${ }^{\star}$
}

\begin{abstract}
We report three-dimensional (3D) direct imaging of complex surface-liquid interfaces by hard X-ray phase contrast tomography as a non-destructive approach for the morphological characterization of surfaces at the micro- and nanoscale in contact with water. Specifically, we apply this method to study the solid-airwater interface in hydrophobic macroporous polymethacrylate surfaces, and the solid-oil-water interface in slippery liquid-infused porous surfaces (SLIPS). Varying the isotropic spatial resolution allows the 3D quantitative characterization of individual polymer globules, globular clusters (porosity) as well as the infused lubricant layer on SLIPS. Surface defects were resolved at the globular level. We show the first application of X-ray nanotomography to hydrated surface characterizations and we anticipate that X-ray nanoscale imaging will open new ways for various surface/interface studies.
\end{abstract}

\section{Introduction}

Surfaces with special wettability attract much research interest due to their great potential to be self-cleaning, ${ }^{\mathbf{1 , 2}}$ cell repellent, anti-fouling or anti-ice coatings., ${ }^{3,4}$ Superhydrophobic ${ }^{5,6}$ and superoleophobic ${ }^{7}$ surfaces have been intensively studied in recent years due to their special liquid repellent properties. Slippery liquid-infused porous surfaces (SLIPS) ${ }^{\mathbf{8}, 9}$ were found to have good anti-bacterial ${ }^{10,11}$ and marine anti-biofouling ${ }^{12}$ properties. However, limitations of the SLIPS were also identified - it was observed that the multiresistant pathogen $P$. aeruginosa was able to form dense biofilms on the slippery poly(butyl methacrylate-co-ethylene dimethacrylate) (BMA-EDMA) surface. ${ }^{\mathbf{1 1}}$ It was

${ }^{a}$ Institute for Photon Science and Synchrotron Radiation (IPS), Karlsruhe Institute of Technology (KIT), D-76344 Karlsruhe, Germany. E-mail: lukas.helfen@kit.edu; Tel: +49721608 26288

${ }^{b}$ European Synchrotron Radiation Facility (ESRF), F-38043 Grenoble, France. Tel: +33 476882558

${ }^{c}$ Institute of Toxicology and Genetics (ITG), Karlsruhe Institute of Technology (KIT), D-76344 Karlsruhe, Germany. E-mail: pavel.levkin@kit.edu

${ }^{d}$ Freiburg Materials Research Center (FMF), University of Freiburg, D-79104 Freiburg, Germany

${ }^{e}$ Department of Applied Physical Chemistry, University of Heidelberg, Im Neuenheimer Feld 253, 69120 Heidelberg, Germany

$\uparrow$ Electronic supplementary information (ESI) available: Details about X-ray phase contrast imaging, X-ray projection microscopy layout, sample geometries, radiation dose calculation, the comparison of phase retrieved and non-phase-retrieved reconstructions and two movies showing the $3 \mathrm{D}$ morphology of the porous BMA-EDMA surface and the slippery BMA-EDMA surface, respectively. See DOI: 10.1039/c3sm52604f

\$ Present address: Institute of Functional Interfaces, Karlsruhe Institute of Technology, 76344 Eggenstein-Leopoldshafen, Germany. also detected that the sporelings of the alga $U$. linza were able to attach to the slippery polymer substrate. ${ }^{12}$

To gain a better understanding of the special wettability and cell repellent properties of these surfaces, methods for direct 3D visualization of complex solid-liquid or solid-air-liquid interfaces at the micro- and nanoscale, under standard ambient pressure and temperature, are needed..$^{13-17}$ To date, visualization of such interfaces in the case of superhydrophobic surfaces has only been reported recently using confocal laser scanning

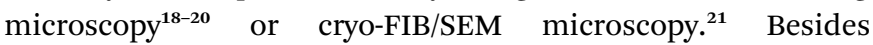
methodical advantages, both methods exhibit limitations concerning either the attainable spatial resolutions or maximum $3 \mathrm{D}$ volume dimensions. In addition, artifacts can be introduced due to the staining (fluorescent dyes) or the freezing processes.

Here we report on 3D in situ imaging of surfaces and complex interfaces in their native states using hard X-ray nanotomography. X-ray tomography with sub-micron resolution is increasingly available at modern synchrotron radiation facilities worldwide. The high penetration depth of X-rays allows the examination of relatively large volumes. The phase sensitivity of partially coherent X-rays opens access to low-density materials. In combination with X-ray microscopy, 3D tomography can be realized with high spatial resolution down to a few tens of nanometers.

Absorption contrast imaging with hard X-rays works well for high- $Z$ elements ( $Z$ is the atomic number) yet produces only weak contrast for materials of low- $Z$ elements. X-ray phase contrast based imaging techniques can be up to $10^{3}$ times more sensitive to weak density variations than the absorption based technique. ${ }^{22}$ Thus, X-ray phase tomography may turn out to be ideal for (super)hydrophobic or slippery microporous surfaces 
which mainly contain light elements like carbon (C), hydrogen $(\mathrm{H})$, oxygen $(\mathrm{O})$ or fluorine $(\mathrm{F})$.

In the present work, we employ hard X-ray holotomography ${ }^{23}$ to investigate hydrophobic porous BMA-EDMA ${ }^{24,25}$ as well as SLIPS prepared using the BMA-EDMA surfaces ${ }^{8,11}$ We demonstrate 3D nanoscale imaging of the solid-air-water interface in the case of non-infused BMA-EDMA surfaces and the solid-oilwater interface in the case of slippery BMA-EDMA surfaces. The measurements were performed in the fully natural state under standard ambient conditions and in mm-thick water environment (results of samples in an air environment are depicted in the ESI $\dagger$ ). Compared to Luo et al. ${ }^{18}$ and Rykaczewski et al.,${ }^{21}$ our imaging approach is faster and non-destructive, and does not require elaborate sample preparation such as sectioning or polishing. The use of a nano-focused X-ray beam in a conic beam projection geometry allows a flexible way to access either nano-resolution (down to $50 \mathrm{~nm}$ pixel size) or a large field of view (FOV) $\left(\sim 0.7 \times 0.7 \mathrm{~mm}^{2}\right)$.

The imaging methods applied in this work are not limited to microporous BMA-EDMA surfaces and slippery BMA-EDMA surfaces, but are beneficial for general micro- and nanoscale study of polymers and their behaviour at multiple-phase interfaces, and are even applicable to samples that require in operando environments.

\section{Materials and methods}

\subsection{Materials preparation}

2.1.1 Preparation of a porous poly(butyl methacrylateethylene dimethacrylate) (BMA-EDMA) surface. The polymerization mixture contains butyl methacrylate (BMA) (20 wt\%), ethylene dimethacrylate (EDMA) (30 wt\%), 1-decanol (50 wt\%), and 2,2-dimethoxy-2-phenylacetophenone (DMPAP) (1 wt\%, with respect to monomers). The polymerization mixture was injected into a mold made of two glass or PMMA substrates, which were separated by two thin strips of Teflon (American Durafilm Co.) with a thickness of $50 \mu \mathrm{m}$. The substrates were then fixed with multiple clamps and put under a UV-lamp (OAI, San Jose, CA) for 15 min of radiation. The intensity of the UV light used was $12 \mathrm{~mW} \mathrm{~cm}$ ch $^{-2}$. After irradiation, the mold was carefully opened with a scalpel. Most of the polymer stuck to the upper substrate, while on the bottom substrate there was only a very thin polymer layer left. The polymer surface on the upper substrate was ready for use after washing extensively with ethanol, immersing in ethanol overnight and then drying with a nitrogen gun. ${ }^{24-26}$

Two groups of porous BMA-EDMA polymer surfaces have been prepared. One group was prepared on microscope cover slip (transparent hydrolytic class 1 glass no. 2, Menzel-Gläser, $25 \mathrm{~mm}$ (width) $\times 60 \mathrm{~mm}$ (length) $\times 0.2 \mathrm{~mm}$ (thickness)) substrates, and the other group on polymethylmethacrylate (PMMA, PLEXIGLAS $®$ XT, $25 \mathrm{~mm}$ (width) $\times 75 \mathrm{~mm}$ (length) $\times$ $1 \mathrm{~mm}$ (thickness)) substrates. All were prepared 48 hours before the actual X-ray imaging experiments.

2.1.2 Preparation of a slippery BMA-EDMA surface. In order to make slippery porous surfaces, an excess amount of perfluoropolyalkylether (PFPE) liquid (Dupont Lubricants
Table 1 A summary of sample types, environment and imaging resolutions

\begin{tabular}{lllll}
\hline$\#$ & Description & Environment & Substrate & Pixel resolution \\
\hline 1 & BMA-EDMA & In water & Glass & $320 \mathrm{~nm}$ \\
2 & BMA-EDMA & In water & Glass & $50 \mathrm{~nm}$ \\
3 & Slippery BMA-EDMA & In water & PMMA & $320 \mathrm{~nm}$ \\
4 & Slippery BMA-EDMA & In water & PMMA & $50 \mathrm{~nm}$ \\
5 & Slippery BMA-EDMA & In water & Glass & $50 \mathrm{~nm}$ \\
6 & Slippery BMA-EDMA & In air & PMMA & $320 \mathrm{~nm}$ \\
7 & Slippery BMA-EDMA & In air & PMMA & $100 \mathrm{~nm}$ \\
8 & Slippery BMA-EDMA & In air & Glass & $320 \mathrm{~nm}$ \\
9 & Slippery BMA-EDMA & In air & Glass & $100 \mathrm{~nm}$
\end{tabular}

Krytox® 103 GPL Oil) was applied on the porous BMA-EDMA surfaces (Fig. S1 in the ESI $\dagger$ ). The surfaces were then tilted at an angle of around $20^{\circ}$ for approximately 2 hours to remove the unbound lubricant from the surface. The water contact angle (WCA) measurements showed that the static WCA decreased from $\sim 133 \pm 3^{\circ}$ on the BMA-EDMA surface to $\sim 114 \pm 2^{\circ}$ on the slippery BMA-EDMA surface (detailed WCA measurements and comparison of non-infused and slippery surfaces are found in ref. 11). The lubricant infusing process was only performed 4 hours before they were scanned with X-rays. The sample list, the corresponding sample environment and pixel resolution used in the imaging experiment are summarized in Table 1.

\subsection{Hard X-ray phase contrast tomography}

2.2.1 Phase contrast imaging. When an X-ray beam penetrates an object, the X-ray waves do not only undergo attenuation by absorption but also phase shifts of the X-ray wavefront, in dependence on the density distribution of the object. In the last decade, several phase-sensitive methods have been developed (more details in Section 2 in the ESI†).

In our paper, propagation-based imaging (PBI) has been employed. ${ }^{23,27-31}$ PBI uses coherence properties of X-ray beams available at modern synchrotron radiation sources. By allowing the transmitted beam to propagate in free space, the phase distortion induced by the object develops into intensity modulations (Fresnel diffraction patterns) at the detector plane (Fig. 1(a))..$^{32}$

X-ray holotomography is used to measure these Fresnel intensity patterns at multiple propagation distances between the sample and the detector plane, and to solve the inverse problem, known as phase retrieval, ${ }^{33}$ that the phase distortion can be retrieved as a so-called phase map (Fig. 1(b)). The phase map represents a projection through the refractive index decrement distribution $(\delta(x, y, z))$ in the object. Therefore, subsequent tomographic reconstruction from the retrieved phase maps of all angles of a tomographic scan will yield the 3D distribution of $\delta(x, y, z)$ in the object, being proportional to the local mass density $\delta \approx 1.36 \times 10^{-6} \rho\left[\mathrm{g} \mathrm{cm}^{-3}\right] \lambda^{2}[\AA]$ for light elements. ${ }^{34}$

2.2.2 Experimental. The holotomography experiments were carried out on the nanoimaging endstation ID22 at the European Synchrotron Radiation Facility (ESRF). A 


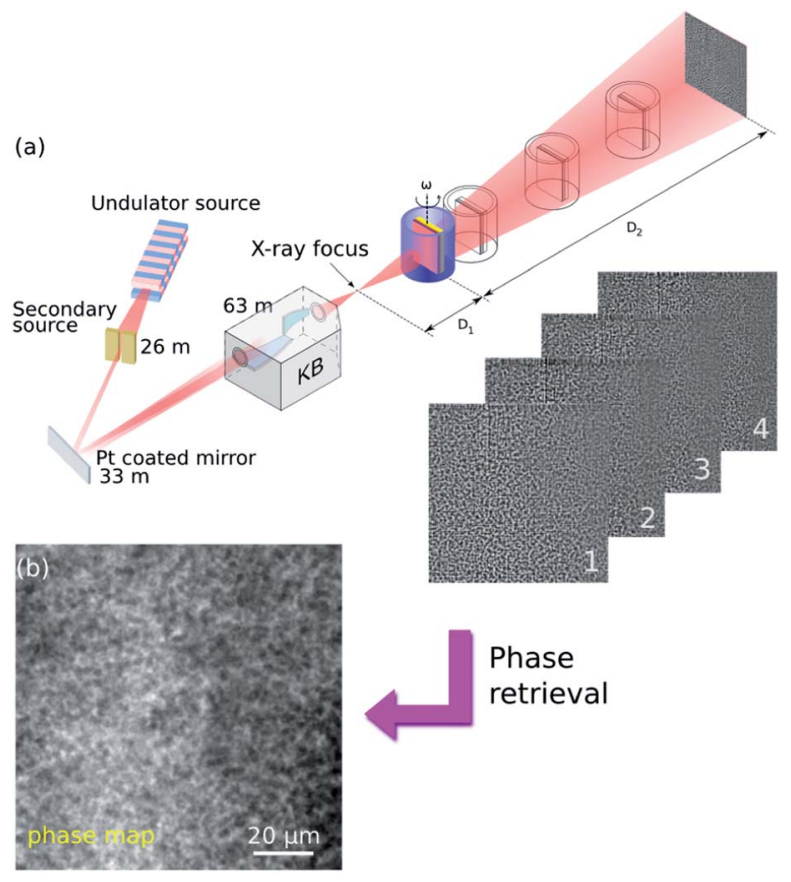

Fig. 1 Principle of holotomography. (a) Sketch of the experimental setup at ESRF beamline ID22NI. (b) Radiographs were recorded at four focus-to-sample distances over a complete tomographic scan with 1199 projection angles. The 4 images were used to reconstruct the phase shift at each angle (phase retrieval). The retrieved phase maps were then used in a tomographic reconstruction step to reconstruct the 3D refractive index decrements of a specific region of interest (ROI) in the sample.

monochromatic X-ray beam $\left(\Delta E / E \simeq 10^{-2}\right.$, pink mode $)$ at an energy of $E=29.6 \mathrm{keV}(\lambda \simeq 0.4 \AA)$ was used. The X-ray beam was focused down to a spot size of approximately $50 \times 50 \mathrm{~nm}^{2}(H \times$ $V$, FWHM) using multilayer coated Kirkpatrick-Baez (KB) optics. ${ }^{35-37}$ The specimen was then placed downstream of the KB focus and magnified onto a camera (the ESRF in-house development FReloN $2 \mathrm{k}^{38}$ ) which uses a charge-coupled device (CCD) with a $2048 \times 2048$ pixel array. The CCD camera is used in combination with an optical microscope system, ${ }^{39,40}$ coupled to a $24 \mu \mathrm{m}$ thick LSO:Tb (terbium doped lutetium-oxyorthosilicate, $\mathrm{Lu}_{2} \mathrm{SiO}_{5}$ ) scintillator, which is optimized for high resolution imaging and provides an effective pixel size of $0.756 \mu \mathrm{m}$.

Employing a slightly divergent cone-beam projection geometry, different magnification factors can be achieved by moving the sample relative to the focal spot (distance $D_{1}$ ) while keeping the detector position fixed at $D_{1}+D_{2}=704.9 \mathrm{~mm}$ (Fig. 1). ${ }^{41}$ The concept enables one to benefit from either a zoom-in detail of the ROI with extremely high resolution or a large FOV, capturing the full-field information in the sample. The geometric magnification factor is calculated from the ratio of the focus-todetector $\left(D_{1}+D_{2}\right)$ and focus-to-sample $\left(D_{1}\right)$ distances $M=\left(D_{1}+\right.$ $\left.D_{2}\right) / D_{1}$. Combined with the large relative propagation distances (sample-to-detector $D_{2}$ ), this so-called projection microscopy provides highly sensitive propagation-based phase contrast images with variable high magnifications leading to spatial resolutions much better than the detector pixel size. In principle, the resolution is then limited by the size of the X-ray focus. In our experiment we employed two scenarios for each type of sample:

(i) $320 \mathrm{~nm}$ pixel size: low resolution scanning accompanied by a large FOV of $650 \times 650 \mu \mathrm{m}^{2}$. One tomographic scan was taken at a fixed sample position $D_{1}=299.1 \mathrm{~mm}$.

(ii) $50 \mathrm{~nm}$ pixel size: high resolution holographic scans to zoom into a much smaller region for detailed visualization. FOV: $102.40 \times 102.40 \mu^{2}$. Four different focus-to-sample distances $D_{1}=\{46.7,47.7,51.7$ and 61.7$\} \mathrm{mm}$ were used in order to cover as much of the Fourier domain as possible (Fig. 1). At each distance a complete tomographic scan was performed. The first distance corresponding to the highest magnification will determine the final pixel size $(100 \mathrm{~nm}$ pixel size is depicted in the ESI $\dagger$ ).

In order to achieve suitable X-ray transmission for our samples, all specimens prepared were cut into pieces of $5 \mathrm{~mm}$ (width) $\times 25 \mathrm{~mm}$ (length). Then the sample fixing using a simple two screw holder was mounted onto an air bearing rotary stage on which tomographic scans were performed (Fig. S2 in the ESI $\dagger$ ). Samples that were scanned in a water environment were kept within cylindrical tubes. The tubes are made of polypropylene (PP) (Ø $8 \mathrm{~mm}, 1 \mathrm{ml}$ Rotilabo®-sample vials H302.1, with tightly fitting caps made of polyethylene (PE)) and were filled with distilled water (Fig. S3 in the ESI†). For a single tomogram, images were recorded at 1199 angularly equidistant positions over a $360^{\circ}$ rotation. The exposure time for each projection was optimized to $0.2 \mathrm{~s}$ to achieve a balance between a good image signal-to-noise ratio (SNR) and a reasonable dose delivered to the sample (Section 3.3 in the ESI†). Taking into account the acquisition time of reference images (20 references were taken between every 400 projections) and dark current images, the scan duration of one complete $3 \mathrm{D}$ tomogram is around $11 \mathrm{~min}$.

The recorded images were not directly used for standard tomographic reconstruction, but allowed the calculation of phase maps via established phase retrieval algorithms. In scenario (i) phase maps were calculated using a single-distance non-iterative phase retrieval algorithm. The algorithm is originally derived based on the assumption that the object consists of a single homogeneous material but varying density. However it can also be applied under more relaxed conditions, e.g. when the density varies only slightly or mostly light elements are present, which is the case in our application. ${ }^{42,43}$ In scenario (ii), all images were aligned and magnified according to the first distance image. At each projection angle the four-distance image set was used to retrieve the phase using a least square optimization based algorithm. ${ }^{23}$ The approach assumes the slowly varying phase (SVP) situation and a linear approximation between the intensity and phase can be made. ${ }^{44}$ Then the phase is retrieved by least square minimization of the measured intensity and the SVP approximation. ${ }^{45,46}$ Finally, for both scenarios the retrieved phase maps were fed into tomographic reconstruction software (ESRF PyHST software package) ) $^{47,48}$ based on the filtered backprojection algorithm. The resulting image corresponds to the $3 \mathrm{D}$ distribution of the refractive index decrement $\delta(x, y, z)$ in the object. 


\section{Results and discussion}

Samples were scanned as listed in Table 1. For all samples, we adhered to imaging procedures that comply with the aforementioned two scenarios. In the following, results of the uncoated surfaces (\#1, \#2) and slippery surfaces (\#3, \#4) are discussed separately. Results of \#5-\#9 are shown in the ESI $\dagger$.

\subsection{Results of microporous BMA-EDMA surfaces}

For the non-infused porous BMA-EDMA surface a full volume that contains $2048 \times 2048 \times 2048$ voxels was reconstructed using a voxel size of $320 \mathrm{~nm}$ (sample \#1, Fig. 2). From the reconstruction, we distinguish the polymer globules (black part in the image), air (white part), water (gray part) and glass substrate (deep black part). We observe that air pockets were well preserved in the interior of the polymer and water could not penetrate into the pores because of the hydrophobicity of the polymer. This effect is known as the Cassie-Baxter state of superhydrophobic surfaces where air is trapped inside porous or rough hydrophobic materials under water. By image analysis the mean thickness of the polymer was measured to be $51.5 \mu \mathrm{m}$. A surface porosity of $\sim 43.5 \%$ was also determined by volume segmentation methods.

In the magnified image Fig. 2(b), two probe lines of $64 \mu \mathrm{m}$ length were chosen and their respective gray value profiles were plotted in Fig. 2(c). It is noted that the magenta arrow line passes through a surface "defect" region, while the green arrow line traverses a defect-free surface. As a result, it is observed that in the magenta plot the "defect" region exhibits smooth gray value variations between the gray levels of water and polymer globules, which means the region was wetted due to the presence of defects near the superficial layer of the polymer.
Table 2 Properties of the materials in the sample ${ }^{a}$

\begin{tabular}{lll}
\hline Materials & Chemical formula & Density $\left(\mathrm{g} \mathrm{ml}^{-1}\right)$ \\
\hline BMA-EDMA & $\mathrm{C}_{m+9 n} \mathrm{H}_{12 m+12 n} \mathrm{O}_{2 m+4 n}(m: n=2: 3)$ & $\sim 1.09$ \\
Krytox® PFPE & $\mathrm{C}_{3 n+2} \mathrm{~F}_{6 n+6} \mathrm{O}_{n}$ & $1.8-1.9$ \\
Water & $\mathrm{H}_{2} \mathrm{O}$ & 1.0 \\
Air & $78.1 \% \mathrm{~N}_{2}, 21.9 \% \mathrm{O}_{2}$ & 0.001 \\
Glass & $72.2 \% \mathrm{SiO}_{2}, 14.3 \% \mathrm{Na}_{2} \mathrm{O}$ & 2.4 \\
PMMA & $\left(\mathrm{C}_{5} \mathrm{O}_{2} \mathrm{H}_{8}\right)_{n}$ & 1.18
\end{tabular}

${ }^{a}$ The chemical formula of BMA-EDMA was described by the $2: 3$ mass ratio of BMA $\left(\mathrm{C}_{8} \mathrm{H}_{14} \mathrm{O}_{2}\right)$ and EDMA $\left(\mathrm{C}_{10} \mathrm{H}_{14} \mathrm{O}_{4}\right)$. The density of BMAEDMA has been determined by weighing the known volume of monomers of the polymerization mixture. The density of Krytox ${ }^{\circledR}$ PFPE oil was taken from DuPont ${ }^{\mathrm{TM}}$ Krytox ${ }^{\circledR}$ Performance Lubricants Data Sheets. ${ }^{49}$ The other densities reported in the table are tabulated values in the XOP DABAX database..$^{50}$

However, in the green plot a big gray level jump is seen at the water-surface interface rather than a slow gray value variation. It implies that where the hydrophobic surface has no defects, it immediately provides strong hydrophobicity. Both arrow lines ended in the glass substrate, where the gray level reached its highest value. The observation is consistent with the relative density distribution of different materials (Table 2).

Fig. 3 shows the $50 \mathrm{~nm}$ high resolution holotomographic reconstruction of the BMA-EDMA surface. The sample only fills half the FOV as the surface has been put in the center of rotation for this tomographic scan. With the smaller FOV in this high resolution image, fine features could be much better resolved compared to the low resolution result. Various overlaps of features appear in Fig. 2, in the high resolution volume we were able to recognize individual polymer globules in 3D. Using a 3D voxel counter tool, ${ }^{51}$ the mean diameter of a single globule was measured to be $1.6 \mu \mathrm{m}$.
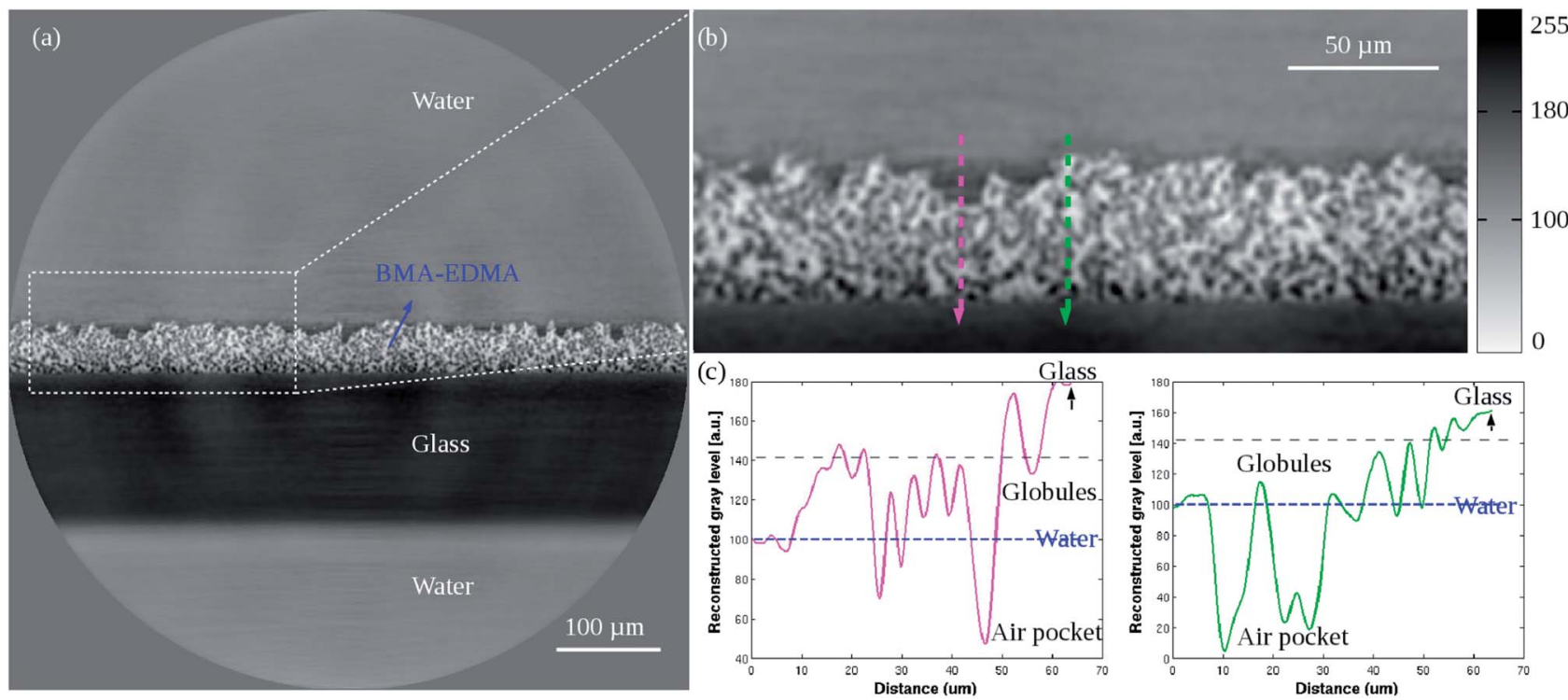

Fig. 2 (a) Low resolution reconstructed 2D cross-section of the porous BMA-EDMA surface on a glass substrate (sample \#1). Pixel size $0.32 \mu \mathrm{m}$. (b) Zoom-in image of the white dashed selection indicated in (a). (c) Profile plots corresponding to the two directional lines (magenta and green) indicated in (b). The horizontal blue dashed line in the plots indicates the gray level of $\mathrm{H}_{2} \mathrm{O}$. The reconstructed gray level qualitatively represents the local mass density of different materials shown in Table 2. Because the sample consists of more than one single material, simple offset corrections were applied to the data based on the known densities of water and air within the sample. 


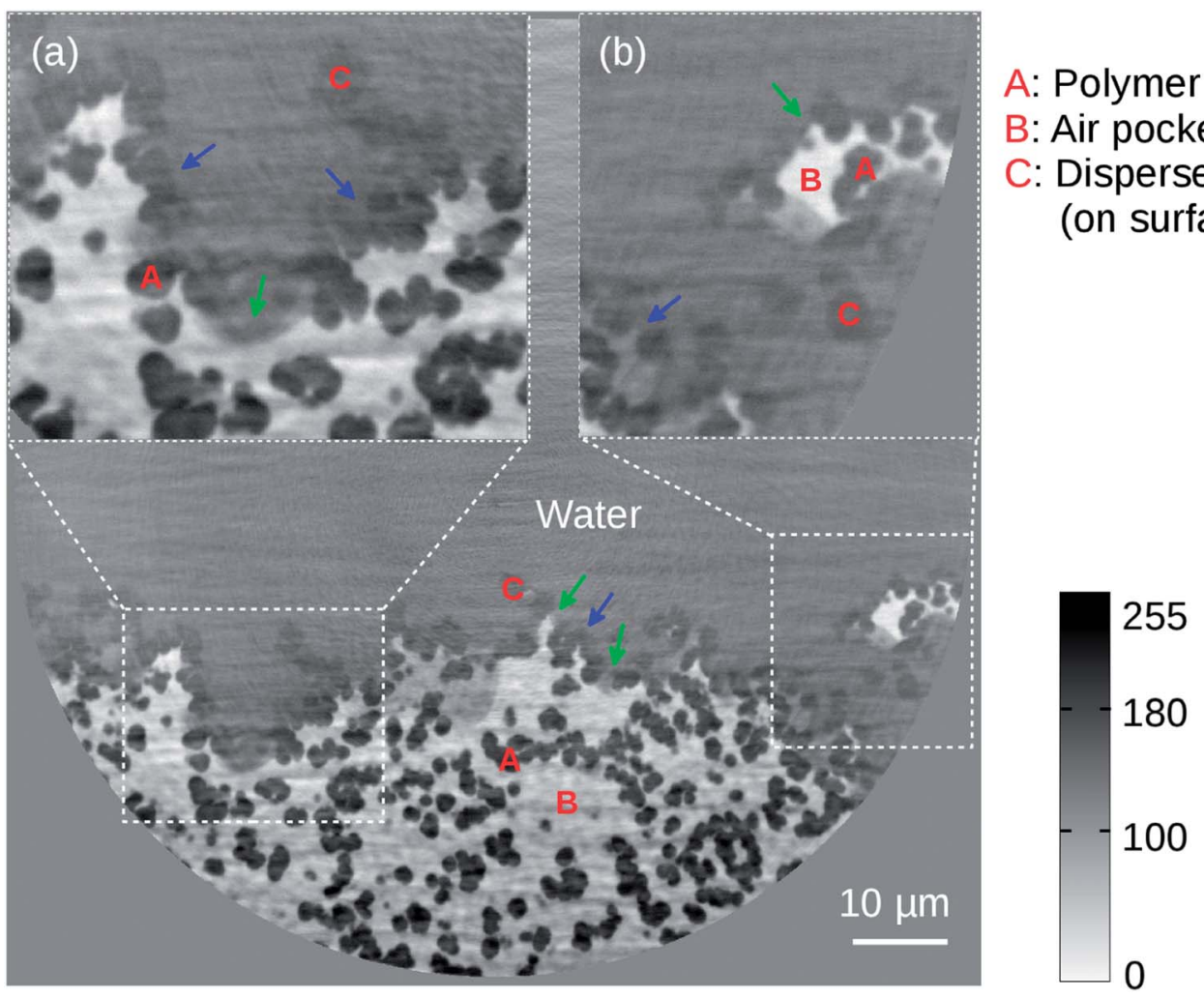

Fig. 3 High resolution reconstructed 2D cross-section of the microporous BMA-EDMA surface on a glass substrate (sample \#2). Effective pixel size was $50 \mathrm{~nm}$. Insets (a) and (b) are zoom-in images of the white dashed selections. Red color labels A, B and C denote polymer globules, air pockets and dispersed globules, respectively. Air was trapped by the structured BMA-EDMA polymer. The coexistence of the Wenzel state (blue arrows) and Cassie-Baxter state (green arrows) when water interacting with the rough surface was observed. A volume of $\sim 0.1 \times 0.05 \times 0.01$ $\mathrm{mm}^{3}$ was rendered in 3D via gray level segmentation (Fig. 6(a)).

As can be seen in the image, three phases that are polymer globules (black part), air (white part) and water (gray part) can be distinguished clearly. We found that the air pockets are efficiently trapped at places where polymer globules are highly concentrated (e.g. region A in the figure). Material defects exist on the superficial layer, normally accompanying globules that are dispersively distributed (region $\mathrm{C}$ in the figure, low concentration). These dispersed globules only appear near the surface, separated from the bulk material and mostly exist at regions where defects are. The most significant difference to the globules in the bulk is that they do not form any porous networks and thus are not able to immobilize any air bubbles. As we clearly observe water somehow fills these "defect" regions. It confirms the observation found in the low resolution image why the gray level of the "defect" area is intermediate between the gray level of water and polymer. These areas comprise a composite of dispersed polymer globules and water. Since both features are not resolvable by the low resolution image the gray level of the defect region showed an average of both components. In contrast, the good surface area which exhibits waterpolymer-air phases shows immediate water-repellent properties, which agrees well with the low resolution observation (Fig. 2(c)).

Based on the above findings we refer to the two existing models that explain the hydrophobicity of a porous surface, namely the Wenzel state ${ }^{14}$ or the Cassie-Baxter state. ${ }^{15}$ In the Wenzel state, water is in contact with the surface and the hydrophobicity originates from the increased surface area of the rough surface. In the Cassie-Baxter state, the trapped air between the rough surface and water is responsible for the hydrophobicity. ${ }^{52}$ As seen in Fig. 3, some of the pores of the BMA-EDMA surface were filled with water while some of the pores were occupied by trapped air pockets, suggesting the coexistence of the Wenzel state and Cassie-Baxter state on the highly hydrophobic BMA-EDMA surface and agreeing with similar observations found in other studies. ${ }^{18,53}$

\subsection{Results of the slippery BMA-EDMA surface}

A volume of slippery BMA-EDMA surface containing $2048 \times$ $2048 \times 2048$ voxels was reconstructed with a voxel size of 320 nm (sample \#3, Fig. 4). The polymer globules (white part), PFPE fluid (dark gray), water (gray) and PMMA substrate (gray) can be clearly recognized. Comparing with Fig. 2(a), a smooth homogeneous layer covering the top of the polymer surface - the infused PFPE liquid, can be observed. The pores in the bulk material were completely filled with the PFPE liquid instead of air. The surface of the coated PFPE liquid layer was not absolutely flat but followed the irregular polymer surface, as can be seen in the zoom-in image Fig. 4(b). 

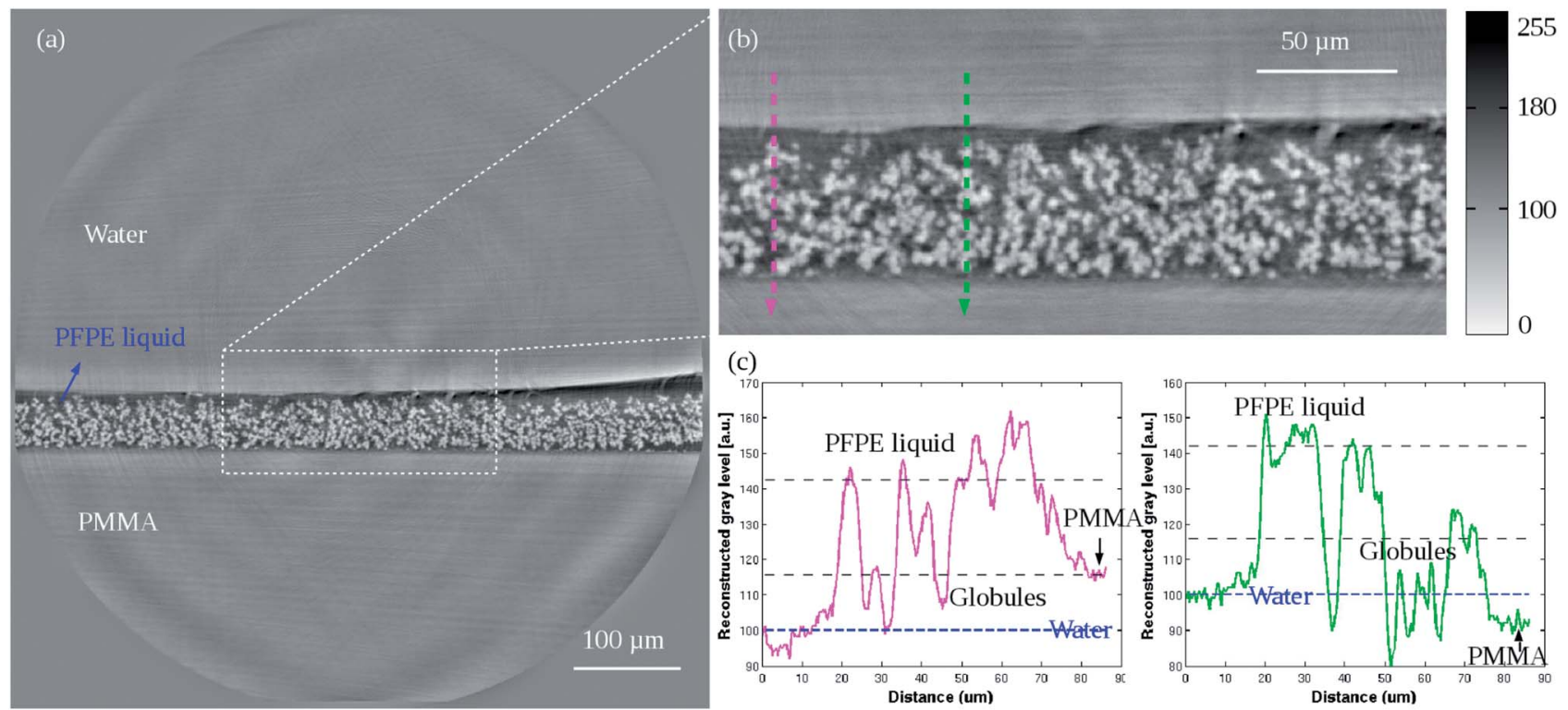

Fig. 4 (a) Low resolution reconstructed 2D cross-section of the slippery BMA-EDMA surface on a PMMA substrate (sample \#3). Pixel size 0.32 $\mu \mathrm{m}$. (b) Zoom-in image of the white dashed selection indicated in (a). (c) Profile plots corresponding to the two directional lines (magenta and green) indicated in (b). The horizontal blue dashed line in the plots point out the gray level of $\mathrm{H}_{2} \mathrm{O}$. The data were corrected and normalized according to the knowledge of the known density of $\mathrm{H}_{2} \mathrm{O}$. Some bias still exists due to big phase jump artifacts that occurred at interfaces and artifacts that were caused by local tomography. But the overall statistical results were qualitatively consistent with the given densities in Table 2 .

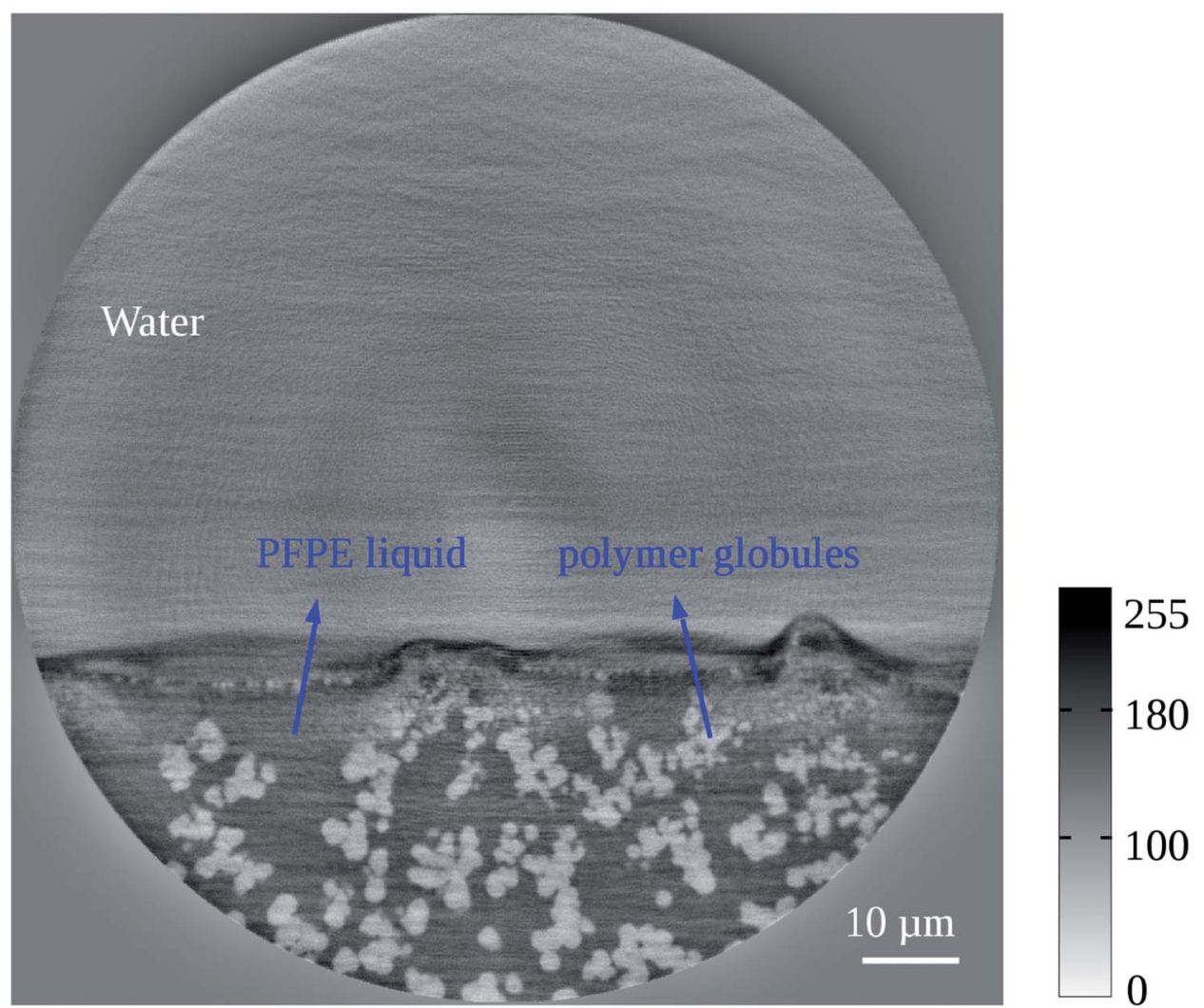

Fig. $550 \mathrm{~nm}$ high resolution reconstructed 2D cross-section of the slippery BMA-EDMA surface (sample \#4). The details of the polymer microstructures correspond to the results of the uncoated BMA-EDMA surface in Fig. 3. However, the air phase that exists in Fig. 3 completely disappeared here and was replaced by the infused PFPE liquid. The liquid was held in place by the rough surface. Water was not anymore able to penetrate into the "defect" area but was already directly repelled by the liquid layer. Because the SVP condition was not completely satisfied in the sample, statistical uncertainty exists in the reconstruction and caused some strong artifacts at the interfaces. 

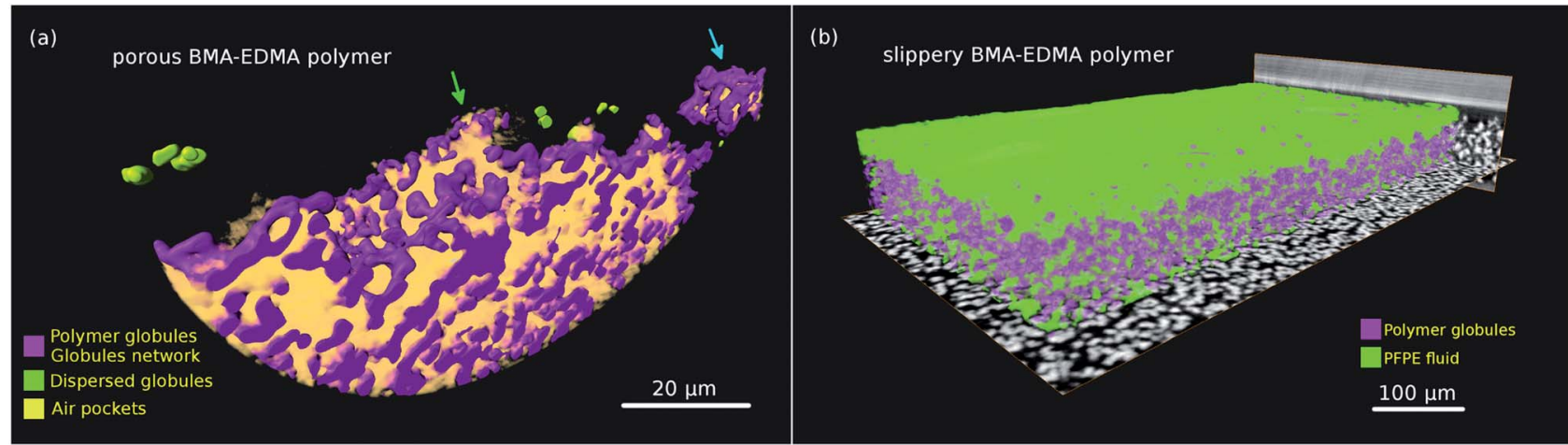

Fig. 6 (a) Volume rendering of the local mass density of the porous BMA-EDMA surface, voxel size $50 \mathrm{~nm}$ (sample \#2). Blue and green arrows indicate Wenzel and Cassie-Baxter states, respectively. (b) Rendering of the slippery BMA-EDMA surface, voxel size $0.32 \mu \mathrm{m}$ (sample \#3). Viewing and analysis of the volumes were performed using the commercial 3D visualization software Amira ${ }^{\circledR} .{ }^{54}$ Two corresponding 3D movies are shown in the ESI†.

Two arrow lines were drawn spanning a length of $86.4 \mu \mathrm{m}$ (Fig. 4(b)) and their gray scale distributions along the paths were quantified in (c). As seen from both plots, the PMMA has an average comparable gray level with that of water $\left(\mathrm{H}_{2} \mathrm{O}\right)$. This is due to the similar densities of both substances (Table 2). By image processing on arbitrarily selected volumes the mean thickness of the pure polymer and the slippery BMA-EDMA surface were measured to be $\sim 49.6 \mu \mathrm{m}$ and $\sim 53.6 \mu \mathrm{m}$, respectively. Hence we obtained a measured average thickness of $4.0 \mu \mathrm{m}$ for the infused PFPE liquid layer. A 3D volume rendering of the slippery BMAEDMA surface is presented in Fig. 6(b) (sample \#3). We can see that the oil did thoroughly diffuse into the BMA-EDMA polymer pores.

It is noted that in some areas near the lubricant interfaces, there are ring and radial stripes (Fig. 4(a and b)). These are reconstruction artifacts caused by the relatively large phase jump between water, PFPE oil and BMA-EDMA polymer interfaces (Table 2). Because the SVP assumption for the phase retrieval was not completely satisfied the accuracy on calculating the material boundaries is reduced. Some extremely bright and dark stripe artifacts at the right border of the interfaces are also seen in Fig. 4(a). They were possibly induced by the total reflection of the X-ray beam in some of the projections because of the flat and smooth sample surface. During tomographic scans, when the sample surface rotated to angles that make it almost parallel to the X-ray beam direction, X-ray reflection could induce such undesirable effects.

A $50 \mathrm{~nm}$ pixel size reconstructed 2D section of the slippery BMAEDMA surface is shown in Fig. 5 (sample \#4). In the current slice we observe a maximum of 80 pixel $(\sim 4 \mu \mathrm{m})$ fluctuation of the lubricant surface due to the anisotropic pressure imposed by the surrounding water environment. The large phase jump at the water-lubricant interfaces again produced reconstruction artifacts. We still see white and dark streaks about the interface identical to the former observation in the low resolution reconstruction (Fig. 4).

Combining the observations from both low and high resolution visualizations, we can again affirm that the lubricant completely fills the polymer pores as well as the superficial defects, bestowing the surface with water repellent and slippery properties.

\section{Conclusions}

We have demonstrated non-invasive direct 3D imaging of surface-water interfaces on hydrophobic porous BMA-EDMA and slippery BMA-EDMA surfaces with X-ray phase contrast nanotomography. The method delivers isotropic volumetric structural characterization of complex interfaces as well as bulk materials from submicron to nano-scales whilst preserving most of the ambient conditions. These advantages are of central importance to help understand the crucial correlations between surface properties and their physical micro- or nanostructures.

For the uncoated microporous BMA-EDMA surfaces first results yield quantitative information about the microstructure (globules' diameter $\sim 1.6 \mu \mathrm{m})$ and the porosity $(\sim 43.5 \%$ ), consistent with the estimated values. ${ }^{25}$ Three phases involving polymer globules, water and air were clearly visualized in 3D. The observation emphasizes the coexistence of the Wenzel and the Cassie-Baxter states at the interface. For the slippery PFPEinfused BMA-EDMA surfaces we observe that the porous surface is entirely wetted by the liquid. The morphology of the infused PFPE fluid was determined (average thickness $\sim 4.0 \mu \mathrm{m}$ ).

For further studies, we expect that the wetting properties of these surfaces at the submicron scale can be quantified by such direct 3D imaging. In addition in situ investigations, for instance, of the formation of the hydrophobic porous structure during polymerization, can be addressed by time-resolved X-ray nano-imaging. To this end, the proposed full-field magnified phase nanotomography is anticipated to have strong impact on quantitative characterization of interfacial materials, thus opening new ways for a better understanding of the performance of anti-fouling, cell repellent superhydrophobic, superoleophobic and various (bio)functional surfaces.

\section{Acknowledgements}

The authors would like to acknowledge ESRF for provisioning the beamtime (SI-2552). Harald Schade, Hugo Vitoux and Sylvain Laboure are greatly thanked for the help of sample preparations. Yang Yang and Dr Thomas van de Kamp are 
appreciated for the help of volume rendering. Dr Stephen Doyle is thanked for proofreading. Y. C. and J. L. thank the China Scholarship Council for PhD fellowships. P. A. L. and J. L. are grateful to the Helmholtz Association's Initiative and Networking Fund (grant VH-NG-621) and BioInterfaces Programme (BIF, KIT) for financial support.

\section{References}

1 H. Budunoglu, A. Yildirim, M. O. Guler and M. Bayindir, ACS Appl. Mater. Interfaces, 2011, 3, 539-545.

2 J. Zimmermann, F. A. Reifler, G. Fortunato, L.-C. Gerhardt and S. Seeger, Adv. Funct. Mater., 2008, 18, 3662-3669.

3 A. K. Epstein, B. Pokroy, A. Seminara and J. Aizenberg, Proc. Natl. Acad. Sci. U. S. A., 2011, 108, 995-1000.

4 P. Kim, T.-S. Wong, J. Alvarenga, M. J. Kreder, W. E. AdornoMartinez and J. Aizenberg, ACS Nano, 2012, 6, 65696577.

5 A. Lafuma and D. Quere, Nat. Mater., 2003, 2, 457-460.

6 L. Feng, S. Li, Y. Li, H. Li, L. Zhang, J. Zhai, Y. Song, B. Liu, L. Jiang and D. Zhu, Adv. Mater., 2002, 14, 1857-1860.

7 A. Tuteja, W. Choi, M. Ma, J. M. Mabry, S. A. Mazzella, G. C. Rutledge, G. H. McKinley and R. E. Cohen, Science, 2007, 318, 1618-1622.

8 T.-S. Wong, S. H. Kang, S. K. Y. Tang, E. J. Smythe, B. D. Hatton, A. Grinthal and J. Aizenberg, Nature, 2011, 477, 443-447.

9 M. Nosonovsky, Nature, 2011, 477, 412-413.

10 A. K. Epstein, T.-S. Wong, R. A. Belisle, E. M. Boggs and J. Aizenberg, Proc. Natl. Acad. Sci. U. S. A., 2012, 109, 13182-13187.

11 J. Li, T. Kleintschek, A. Rieder, Y. Cheng, T. Baumbach, U. Obst, T. Schwartz and P. A. Levkin, ACS Appl. Mater. Interfaces, 2013, 5, 6704-6711.

12 L. Xiao, J. Li, S. Mieszkin, A. Di Fino, A. S. Clare, M. E. Callow, J. A. Callow, M. Grunze, A. Rosenhahn and P. A. Levkin, ACS Appl. Mater. Interfaces, 2013, 5, 10074-10080.

13 T. Young, Philos. Trans. R. Soc. London, 1805, 95, 65-87.

14 R. N. Wenzel, Ind. Eng. Chem., 1936, 28, 988-994.

15 A. B. D. Cassie and S. Baxter, Trans. Faraday Soc., 1944, 40, 546-551.

16 L. Gao and T. J. McCarthy, Langmuir, 2007, 23, 3762-3765.

17 B. Haimov, S. Pechook, O. Ternyak and B. Pokroy, J. Phys. Chem. C, 2013, 117, 6658-6663.

18 C. Luo, H. Zheng, L. Wang, H. Fang, J. Hu, C. Fan, Y. Cao and J. Wang, Angew. Chem., Int. Ed., 2010, 49, 9145-9148.

19 P. Papadopoulos, X. Deng, L. Mammen, D.-M. Drotlef, G. Battagliarin, C. Li, K. Mllen, K. Landfester, A. del Campo, H.-J. Butt and D. Vollmer, Langmuir, 2012, 28, 8392-8398.

20 P. Papadopoulos, L. Mammen, X. Deng, D. Vollmer and H.-J. Butt, Proc. Natl. Acad.Sci. U. S. A., 2013, 110, 32543258.

21 K. Rykaczewski, T. Landin, M. L. Walker, J. H. J. Scott and K. K. Varanasi, ACS Nano, 2012, 6, 9326-9334.

22 A. Momose, T. Takeda, Y. Itai and K. Hirano, Nat. Med., 1996, $2,473-475$.
23 P. Cloetens, W. Ludwig, J. Baruchel, D. van Dyck, J. van Landuyt, J. Guigay and M. Schlenker, Appl. Phys. Lett., 1999, 75, 2912.

24 P. A. Levkin, F. Svec and J. M. J. Fréchet, Adv. Funct. Mater., 2009, 19, 1993-1998.

25 J. S. Li, E. Ueda, A. Nallapaneni, L. X. Li and P. A. Levkin, Langmuir, 2012, 28, 8286-8291.

26 D. Zahner, J. Abagat, F. Svec, J. M. J. Fréchet and P. A. Levkin, Adv. Mater., 2011, 23, 3030-3034.

27 A. Snigirev, I. Snigireva, V. Kohn, S. Kuznetsov and I. Schelokov, Rev. Sci. Instrum., 1995, 66, 5486-5492.

28 P. Cloetens, R. Barrett, J. Baruchel, J.-P. Guigay and M. Schlenker, J. Phys. D: Appl. Phys., 1996, 29, 133.

29 K. A. Nugent, T. E. Gureyev, D. F. Cookson, D. Paganin and Z. Barnea, Phys. Rev. Lett., 1996, 77, 2961-2964.

30 S. W. Wilkins, T. E. Gureyev, D. Gao, A. Pogany and A. W. Stevenson, Nature, 1996, 384, 335-338.

31 P. Cloetens, W. Ludwig, J. Baruchel, J.-P. Guigay, P. PernotRejmánková, M. Salomé-Pateyron, M. Schlenker, J.-Y. Buffière, E. Maire and G. Peix, J. Phys. D: Appl. Phys., 1999, 32, A145.

32 P. Cloetens, Ph.D. thesis, Vrije Universiteit Brussel and ESRF, 1999.

33 P. Cloetens, W. Ludwig, E. Boller, L. Helfen, L. Salvo, R. Mache and M. Schlenker, Proceedings SPIE: Developments in X-Ray Tomography III, 2002, pp. 82-91.

34 A. Guinier, X-ray diffraction: in crystals, imperfect crystals, and amorphous bodies, Dover Publications, Mineola, NY, 1994.

35 R. Mokso, P. Cloetens, E. Maire, W. Ludwig and J.-Y. Buffiere, Appl. Phys. Lett., 2007, 90, 144104.

36 H. Suhonen, F. Xu, L. Helfen, C. Ferrero, P. Vladimirov and P. Cloetens, Int. J. Mater. Res., 2012, 2, 179-183.

37 G. Martínez-Criado, R. Tucoulou, P. Cloetens, P. Bleuet, S. Bohic, J. Cauzid, I. Kieffer, E. Kosior, S. Labouré, S. Petitgirard, A. Rack, J. A. Sans, J. Segura-Ruiz, H. Suhonen, J. Susini and J. Villanova, J. Synchrotron Radiat., 2012, 19, 10-18.

38 J.-C. Labiche, O. Mathon, S. Pascarelli, M. A. Newton, G. G. Ferre, C. Curfs, G. Vaughan, A. Homs and D. F. Carreiras, Rev. Sci. Instrum., 2007, 78, 091301.

39 T. Martin and A. Koch, J. Synchrotron Radiat., 2006, 13, 180194.

40 P. A. Douissard, A. Cecilia, X. Rochet, X. Chapel, T. Martin, T. van de Kamp, L. Helfen, T. Baumbach, L. Luquot, X. Xiao, J. Meinhardt and A. Rack, J. Instrum., 2012, 7, P09016.

41 P. Bleuet, P. Cloetens, P. Gergaud, D. Mariolle, N. Chevalier, R. Tucoulou, J. Susini and A. Chabli, Rev. Sci. Instrum., 2009, 80, 056101.

42 D. Paganin, S. C. Mayo, T. E. Gureyev, P. R. Miller and S. W. Wilkins, J. Microsc., 2002, 206, 33-40.

43 T. Weitkamp, D. Haas, D. Wegrzynek and A. Rack, J. Synchrotron Radiat., 2011, 18, 617-629.

44 J. P. Guigay, Optik, 1977, 49, 121-125.

45 S. Zabler, P. Cloetens, J.-P. Guigay, J. Baruchel and M. Schlenker, Rev. Sci. Instrum., 2005, 76, 073705. 
46 M. Langer, P. Cloetens, J.-P. Guigay and F. Peyrin, Med. Phys., 2008, 35, 4556-4566.

47 A. Mirone, E. Brun, E. Gouillart, P. Tafforeau and C. Ferrero, Nucl. Instrum. Methods Phys. Res., Sect. B, 2014, DOI: 10.1016/ j.nimb.2013.09.030.

48 S. Chilingaryan, A. Mirone, A. Hammersley, C. Ferrero, L. Helfen, A. Kopmann, T. dos Santos Rolo and P. Vagovič, IEEE Trans. Nucl. Sci., 2011, 58, 1447-1455.

49 DuPont $^{\mathrm{TM}}$ Krytox$®$ Performance Lubricants, Krytox ${ }^{\circledR}$ Data Sheets, 2013, http://www2.dupont.com/Lubricants/en_US/ index.html.
50 M. Sanchez del Rio and R. J. Dejus, Crystal and Multilayer Optics, Proc. SPIE, 1998, 3448, 340-345.

51 C. A. Schneider, W. S. Rasband and K. W. Eliceiri, Nat. Methods, 2012, 9, 671-675.

52 A. Lafuma and D. Quere, Nat. Mater., 2003, 2, 457460.

53 T. Koishi, K. Yasuoka, S. Fujikawa, T. Ebisuzaki and X. C. Zeng, Proc. Natl. Acad. Sci. U. S. A., 2009, 106, 84358440.

54 VSG - Visualization Sciences Group, Amira ${ }^{\circledR}$ - 3D Analysis Software, 2012, http://www.vsg3d.com/amira/overview. 